\title{
Investigation of Chronic Obstructive Pulmonary Disease (COPD) Development in Terms of Urea, Creatinine and Some Blood Parameters in Patients with Allergic Rhinitis
}

\author{
Seha Akduman \\ Pulmonary Medicine, Yeditepe Üniversitesi Koşuyolu Hastanesi, Koşuyolu, İstanbul/Kadıköy \\ İstanbul, TURKEY
}

*Corresponding author - Seha Akduman; akdumanseha@ hotmail.com

Received 01 March 2019;

Accepted 17 March 2019;

Published 20 March 2019

\begin{abstract}
Aim: In this retrospective crossectional study, it was aimed to evaluate the development of COPD in terms of urea, creatinine and some blood parameters in patients with allergic rhinitis. Method: Patients who were admitted to Kadiköy Medicana Hospital between 12.10 .2017 and 12.10.2018 with respiratory complaints and has COPD diagnosis for the first time were evaluated retrospectively. Among the 845 patients admitted to the clinic for a one-year period, 160 patients with the diagnosis of COPD for the first time and 42 patients with a history of allergic rhinitis were subjected. The study group consisted of 63 patients with COPD who were diagnosed as COPD for the first time but without allergic rhinitis and 57 allergic rhinitis patients with no diagnosis of COPD. Findings: CRP, urea, WBC and neutrophil levels were higher in COPD group; creatinine and eisonofil levels were higher in COPD-Allergic rhinitis group and HGB was higher in the allergic rhinitis group. The differences between CRP and urea levels of COPD and allergic rhinitis group were statistically significant $(\mathrm{p}<0.05)$. The urea values were significantly different between COPD + allergic rhinitis and COPD groups ( $p<0.05)$. According to the results of ROC analysis, the value of urea for the COPD + allergic rhinitis group was statistically insignificant ( $>0.05$ ), whereas it was statistically significant for the allergic rhinitis group ( $\mathrm{p}<0.05$ ). Conclusion: In detecting the difference between allergic rhinitis and COPD associated allergic rhinitis, urea levels may have a diagnostic value. An increase in urea in patients with allergic rhinitis may indicate comorbid COPD.
\end{abstract}

Keywords: Allergic rhinitis, blood count, creatinine, COPD, urea, WBC.

\section{Introduction}

Allergic rhinitis (AR) is one of the common pulmonary diseases and associated with asthma and conjunctivitis, and associated with considerable morbidity. AR is characterized by rhinorrea, nasal itching, nasal drainage and nasal congestion. It is common in adults and children, and its prevalence was $14.6 \%$ at 13 and 14 age adolescents ${ }^{[1-5]}$. AR is classified by a) temporal pattern triggered by seasonal allergens, b) symptoms frequency and c) symptom severity ${ }^{[3]}$. AR affects daily activities and reduces sleep quality. IgE mediated mast cell degranulation causes nasal blockage ${ }^{[6-8]}$. $\operatorname{IgE}$ is the most common test, however, it is not themselves sufficient for diagnose. The ultimate standard diagnosis must combine positive history, IgE inflammation related symptoms and presence of $\operatorname{IgE}^{[9]}$. Treatment of $\mathrm{AR}$ includes nasal and oral antihistamines, intranasal glucocortioids and leukotriene-receptor antagonists. AR treatment is associated with benefits for asthma ${ }^{[1,7]}$. In case of a persist symptom, an allergist may be considered $^{[10]}$.

Chronic obstructive pulmonary disease (COPD) is a preventable and treatable common lung disease. COPD may have comorbid with obstructive bronchiolitis, emphysema or combination of both syndromes ${ }^{[11-12]}$. The disease has a slow and debilitating character with physical symptoms and loss of function ${ }^{[13]}$. COPD causes airflow restriction and changes in lung, and for this reason, it is associated with comorbidities and systemic effects ${ }^{[14]}$. Examining blood and serum parameters in AR patients may have diagnostic value for COPD. Thus, it is aimed to investigate the development of COPD in terms of urea, creatinine and some blood parameters in patients with allergic rhinitis in this research. 


\section{Methods}

Patients who were admitted to Kadıköy Medicana Hospital between 12.10 .2017 and 12.10 .2018 with respiratory complaints with for the first time COPD diagnosis were evaluated retrospectively. The study group consisted of patients with a firstly diagnosis of COPD and had a history of allergic rhinitis. The criteria for inclusion and exclusion in the study were as follows:

- First diagnosed with COPD,

- Previously diagnosed with allergic rhinitis,

- Clinical picture and epicrisis do not interfere with research design

In this context, out of the 845 patients admitted to the clinic during a one-year period, 160 patients with COPD for the first time were diagnosed with 42 patients with a history of allergic rhinitis. The study group consisted of 63 patients with COPD who were diagnosed as COPD for the first time but without allergic rhinitis and 57 allergic rhinitis patients with no diagnosis of COPD.
Nominal data were described by frequency analysis and scale parameters were described by mean and standard deviation values. Kolmogorov Smirnov test was applied for the normality distribution of the measurement data before the difference analysis. Independent Samples T-test was used for differences of WBC and HGB parameters which showed normal distsibution. Mann Whitney-U test was used for differences of CRP, urea, creatinin, NEU and EOS parameters which did not show normal distribution.

ROC analysis was used for the diagnostic value of urea parameter which was significant in each group. All analyzes were performed in SPSS 17.0 for Windows package, 95\% confidence interval and 0.05 significance level.

\section{Results}

The distribution of some clinical and demographic parameters of patient groups is given in Table 1.

Table 1: Baseline and clinical parameters of patient groups

\begin{tabular}{|c|c|c|c|}
\hline Parameter & COPD+ Allergic Rhinitis $(n=42)$ & $\begin{array}{l}\text { COPD without Allergic rhinitis } \\
(n=63)\end{array}$ & $\begin{array}{l}\text { Allergic Rhinitis without } \\
\text { COPD }(n=57)\end{array}$ \\
\hline Female, n (\%) & $27(64.3)$ & $25(39.7)$ & 37 (64.9) \\
\hline Male, n (\%) & $15(35.7)$ & $38(60.3)$ & $20(35.1)$ \\
\hline Age, Mean \pm SD & $66.45 \pm 15.20$ & $69.81 \pm 13.52$ & $48.00 \pm 18.96$ \\
\hline CRP & $1.39 \pm 2.64$ & $2.26 \pm 3.96$ & $0.80 \pm 1.33$ \\
\hline Urea & $44.23 \pm 23.99$ & $51.83 \pm 35.13$ & $32.90 \pm 20.51$ \\
\hline Creatine & $0.93 \pm 0.47$ & $0.92 \pm 0.40$ & $0.85 \pm 0.46$ \\
\hline WBC & $7.76 \pm 3.04$ & $8.68 \pm 2.98$ & $7.66 \pm 2.00$ \\
\hline NEU & $5.19 \pm 2.88$ & $6.04 \pm 2.92$ & $4.97 \pm 1.72$ \\
\hline Eos & $0.20 \pm 0.17$ & $0.19 \pm 0.20$ & $0.17 \pm 0.15$ \\
\hline HGB & $13.35 \pm 1.75$ & $13.51 \pm 2.09$ & $13.59 \pm 1.56$ \\
\hline
\end{tabular}

The study included 27 women (64.3\%) and 15 men (35.7\%) from COPD + Allergic Rhinitis group; 25 women (39.7\%) and 38 men (60.3\%) from the COPD group; 37 women $(64.9 \%)$ and 20 men $(35.1 \%)$ were included in the allergic rhinitis group. The mean age of COPD + allergic rhinitis group was $66.45 \pm 15.20$, mean age of COPD group was $69.81 \pm 13.52$ and mean age of allergic rhinitis group was $48.00 \pm 18.96$. CRP, urea, WBC and neutrophil levels were higher in COPD group; creatinine and eisonofil levels were higher in COPD-Allergic rhinitis group and HGB in allergic rhinitis group. The results of the difference analysis for the two comparisons between the groups are given in Table 2 .

Table 2: Difference analysis results between paired groups and $p$ values

\begin{tabular}{|c|c|c|c|}
\hline Parameter & Group 1/Group2 & Group 1/Group3 & Group 2/Group3 \\
\hline $\mathrm{CRP}^{\mathrm{b}}$ & 0.310 & 0.342 & $0.027 *$ \\
\hline Urea $^{b}$ & 0.339 & $0.004 *$ & $0.000 *$ \\
\hline Creatine $^{\mathrm{b}}$ & 0.936 & 0.203 & 0.079 \\
\hline $\mathrm{WBC}^{\mathrm{a}}$ & 0.175 & 0.949 & 0.141 \\
\hline $\mathrm{NEU}^{\mathrm{b}}$ & 0.123 & 0.975 & 0.113 \\
\hline $\mathrm{EOS}^{\mathrm{b}}$ & 0.516 & 0.460 & 0.959 \\
\hline $\mathrm{HGB}^{\mathrm{a}}$ & 0.666 & 0.464 & 0.954 \\
\hline
\end{tabular}

a. Independent Samples T-Test, b. Mann Whitney-U test.

As seen from the table, the difference between CRP and urea levels of COPD and allergic rhinitis group was statistically significant ( $p$ $<0.05$ ). The urea values were significantly different between COPD + allergic rhinitis and COPD groups $(\mathrm{p}<0.05)$. Urea values were the highest in the group first time COPD diagnosed and without allergic rhinitis. While urea was lower in COPD + allergic rhinitis group, it was lowest in only allergic rhinitis group. Accordingly, the results of the ROC analysis for the diagnostic value of urea values were as Scheme 1 . 
Scheme 1: The results of the ROC analysis for the diagnostic value of urea values

ROC Curve

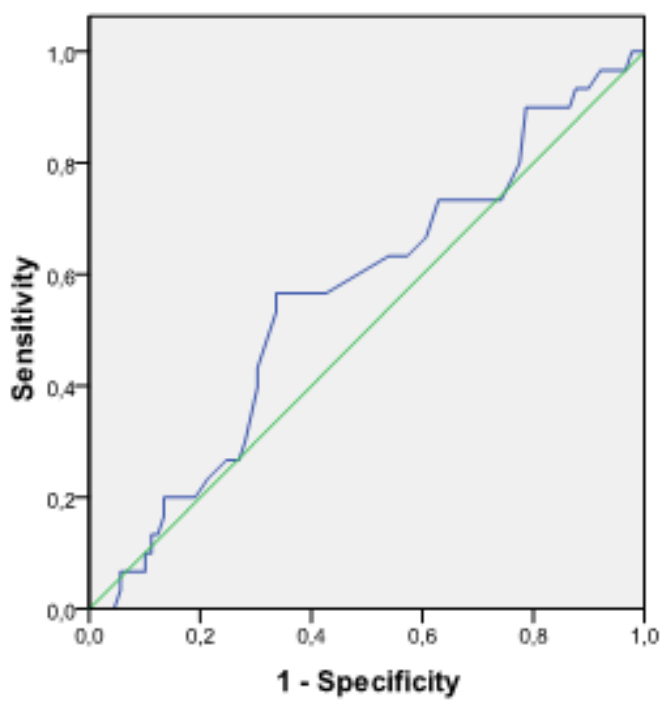

Diagonal segments are produced by ties.
ROC Curve

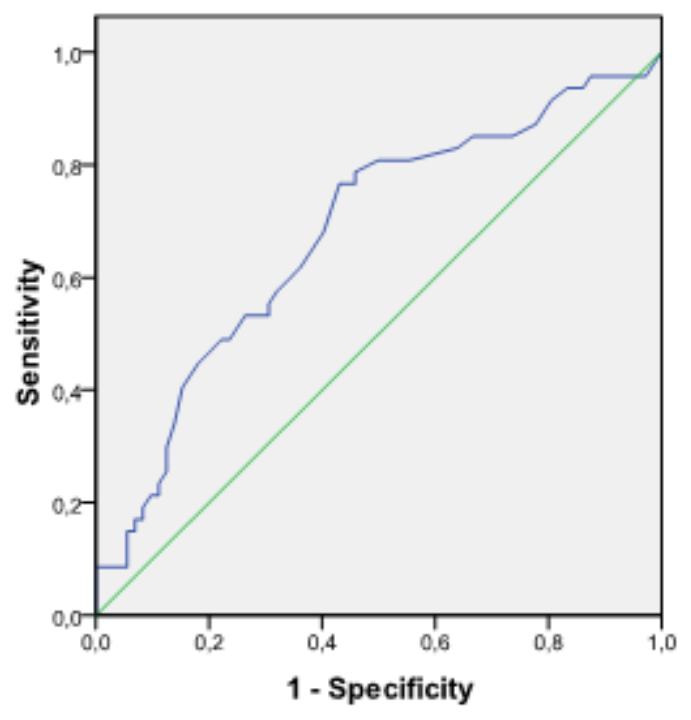

Diagonal segments are produced by ties.

a. $\mathrm{R}^{2}=0.561$ for COPD+ Allergic Rhinitis

b. $\mathrm{R}^{2}=0.678^{*}$ for Allergic Rhinitis

According to the results of ROC analysis, the value of urea for the COPD + allergic rhinitis group was statistically insignificant ( $>>0.05$ ), whereas it was statistically significant for the allergic rhinitis group $(\mathrm{p}<0.05)$.

\section{Discussion}

In the literature, there have been researches reporting gender and age effects on COPD disease ${ }^{[15-17]}$ and allergic rhinitis ${ }^{[18-20]}$. In general, these studies report that COPD appeared at an older age ${ }^{[21-}$ ${ }^{23]}$, whereas allergic rhinitis was reported at an earlier age ${ }^{[24-26]}$. In our study, when the distribution by gender was examined, male gender was the most common in patients diagnosed with COPD rather than allergic rhinitis. On the other hand, in the group without allergic rhinitis and in the group with the first diagnosis of COPD, the males were statistically dominant. When the mean age was examined, the mean age was higher in both COPD groups with and without the diagnosis of allergic rhinitis. On the other hand, the mean age of patients with allergic rhinitis but not COPD was statistically significantly lower.

In the literature, there are studies reporting different values of blood in both $\mathrm{COPD}^{[27-29]}$ and allergic rhinitis cases ${ }^{[30,31]}$. In our study, creatinine and EOS were the highest in the group of patients diagnosed with COPD for the first time and diagnosed as allergic rhinitis. CRP, urea, WBC and NEU were higher in the group with the first diagnosis of COPD but without allergic rhinitis. Only patients with allergic rhinitis had only higher HGB levels. It may be argued that the difference in the average age is effective on this result. On the other hand, when allergic rhinitis is accompanied by COPD, blood values, CRP and urea values may be decreased. According to the results of the difference analysis, no significant difference was found between the urea values of patients with allergic rhinitis who were diagnosed with COPD for the first time and patients without allergic rhinitis. However, in the group with the first diagnosis of COPD and in the group with allergic rhinitis with COPD, the urea levels were statistically higher than those with allergic rhinitis without COPD.

\section{Conclusion}

According to the findings of the study, although age is the most important indicator for the suspicion of COPD in elderly patients who applied to the clinic with the complaint of respiratory tract; the urea value may be an important indicator in patients who have been diagnosed with allergic rhinitis before.

According to the results of ROC analysis, urea values have diagnostic importance in detecting the difference between allergic rhinitis and COPD accompanying allergic rhinitis. Therefore, it is useful to treat patients with allergic rhinitis with a high urea value in the range of normal values according to age group with suspicion of diagnosis of COPD.

\section{Acknowledgment}

The research was conducted by authors own efforts.

\section{Financial support \& Sponsorship}

The research was funded by authors own budget.

\section{Conflicts of interest}

The authors declare that there is no conflict of interest regarding the publication of this paper.

\section{References}

[1] Wheatley LM and Togias A. Allergic Rhinitis. N Engl J Med 2015; 372:456-463. 
[2] Angier E, Willington J, Scadding G, Holmes S and Walker S. Management of allergic and non-allergic rhinitis: a primary care summary of the BSACI guideline. Primary Care Respiratory Journal (2010); 19(3): 217-222.

[3] Seidman et al. Clinical Practice Guideline: Allergic Rhinitis Executive Summary. Head and Neck Surgery 2015, Vol. 152(2) 197-206.

[4] Small P, Keith PK and Kim H. Allergic rhinitis. Allergy, Asthma \& Clinical Immunology, 2018; 14 (Suppl 2):51.

[5] Barr JG, Al-Reefy H, Fox AT and Hopkins C. Allergic rhinitis in children. BMJ 2014; 348:g4153.

[6] Walker SM et al. Immunotherapy for allergic rhinitis. Clinical \& Experimental Allergy, 2011; (41) 1177-1200.

[7] Scadding GK et al. BSACI guidelines for the management of allergic and non-allergic rhinitis. Clinical and Experimental Allergy, 2008; 38, 19-42.

[8] Sin B and Togias A. Pathophysiology of Allergic and Nonallergic Rhinitis. Proc Am Thorac Soc, 2011; Vol 8. pp 106-114.

[9] Skoner DP. Allergic rhinitis: Definition, epidemiology, pathophysiology, detection, and diagnosis. J. Allergy Clin Immunol, 2001; (108)1, 1-7.

[10] Small P and Kim H. Allergic rhinitis. Allergy, Asthma \& Clinical Immunology 2011; 7(Suppl 1):S3

[11] Laraeau SC, Fahy B, Meek P and Wang A. (2019). "Chronic Obstructive Pulmonary Disease (COPD)". Am J Respir Crit Care Med Vol. 199, P1-P2.

[12] Prosser TR and Bolmeier SG. (2008). "Chronic Obstructive Pulmonary Disease". Pharmacotheraphy Self-Assesment Program, 6th Edition.

[13] Landers A, Wiseman R, Pitama S, et al. Severe COPD and the transition to a palliative approach. Breathe 2017; 13: $310-316$.

[14] OHTAC COPD Collaborative. Chronic obstructive pulmonary disease (COPD) evidentiary framework. Ont Health Technol Assess Ser [Internet]. 2012 March;12(2):1-97.

[15] Farooqi N, Carlsson M, Haglin L, Sandström T and Slinde F. (2018). "Energy expenditure in women and men with COPD". Clinical Nutrition ESPEN, 28(1), 171178.

[16] Delgado A, Saletti-Cuesta L, Lopez-Fernandez LA, GilGarrido N and Castillo JDL. (2016). "Gender inequalities in COPD decision-making in primary care". Respiratory Medicine, 114(1), 91-96.

[17] Roberts NJ, Patel IS and Partridge MR. (2016). "The diagnosis of COPD in primary care; gender differences and the role of spirometry". Respiratory Medicine, 111(1), 60-63.
[18] Kang X et al. "Home environment and diseases in early life are associated with allergic rhinitis". International Journal of Pediatric Otorhinolaryngology, 118(1), 47-52.

[19] Chong SN and Chew FT. (2018). Epidemiology of allergic rhinitis and associated risk factors in Asia". World Allergy Organization Journal, Volume 11, 2018, Article D17.

[20] Yonekura S. et al. (2017). "Complementary and alternative medicine for allergic rhinitis in Japan". Allergology International, 66(3), 425-431.

[21] Bowdish DME. (2019). "The Aging Lung: Is Lung Health Good Health for Older Adults?". Chest, 155(2), 391-400.

[22] Kusumastuti S, Rozing MP, Lund R, Mortensen EL and Westendorp RGJ. (2018). "The added value of health indicators to mortality predictions in old age: A systematic review". European Journal of Internal Medicine, 57(1), 7-18.

[23] Roos EW. Et al. (2018). "Asthma and its comorbidities in middle-aged and older adults; the Rotterdam Study". Respiratory Medicine, 19(1), 6-12.

[24] Kou W, Li X, Yao H and Wei P. (2018). "Meta-analysis of the comorbidity rate of allergic rhinitis and asthma in Chinese children". International Journal of Pediatric Otorhinolaryngology, 107(1), 131-134.

[25] Tharpe CA and Kemp SF. (2015). "Pediatric Allergic Rhinitis". Immunology and Allergy Clinics of North America, 35(1), 185-198.

[26] Corren J. (2017). Chapter 8: Allergic Rhinitis and Conjunctivitis. Middleton's Allergy Essentials, 2017, Pages 205-224.

[27] Suissa S, Dell'Aniello S and Ernst P. (2018). "Comparative effectiveness of LABA-ICS versus LAMA as initial treatment in COPD targeted by blood eosinophils: a population-based cohort study". The Lancet Respiratory Medicine, 6(11), 855-862.

[28] Gonçalves I. et al. (2018). "Clinical and molecular markers in COPD”. Pulmonology, 24(4), 250-259.

[29] Couillard S, Larivee P, Courteau J and Vanasse A. (2017). "Eosinophils in COPD Exacerbations Are Associated With Increased Readmissions". Chest, 151(2), 366-373.

[30] Akgedik R and Yağız Y. (2017). "Is Decreased Mean Platelet Volume in Allergic Airway Diseases Associated With Extent of the Inflammation Area?". The American Journal of the Medical Sciences, 354(1), 33-38.

[31] Jordakieva G and Jensen-Jarolim E. (2018). "The impact of allergen exposure and specific immunotherapy on circulating blood cells in allergic rhinitis". World Allergy Organization Journal, 11(1), Article D19. 